\title{
Runnorexia: una revisión sobre la adicción al ejercicio físico en corredores Runnorexia: a review of physical exercise addiction in runners
}

\author{
Joel Manuel Prieto Andreu \\ Universidad Internacional de La Rioja (España)
}

\begin{abstract}
Resumen. Para el sujeto con runnorexia todo puede girar en torno al entrenamiento, lo que podría acarrear modificaciones en sus hábitos, alterando su estilo de vida, alimentación y relaciones sociales. El objetivo de esta revisión consiste en realizar un análisis de los estudios sobre adicción al ejercicio físico en corredores y establecer el perfil del corredor que se encuentra en riesgo de poseer una adicción a salir a correr. La búsqueda de artículos se realizó en las bases de datos Scopus y Web of Science, utilizando combinaciones de las palabras clave: «adicción», «ejercicio físico», «corredores». Se siguieron las directrices de la declaración PRISMA y se analizaron los participantes, instrumentos y resultados de los 12 trabajos seleccionados de acuerdo con los criterios de inclusión/ exclusión, evaluándose el rigor metodológico mediante un sistema de clasificación estructurado. Los resultados indican que la runnorexia podría presentarse en corredores aficionados de larga distancia entre 35 y 45 años con una menor experiencia deportiva, con elevada Adicción Negativa a Correr (ANC) y ansiedad provocada por la necesidad de competir, con búsqueda de reconocimiento social y dominio, con preocupación acerca de sus errores y con unos altos estándares personales.
\end{abstract}

Palabras clave: adicción; ejercicio físico; ejercicio; corredores; correr.

\begin{abstract}
For runnorexic everything can revolve around training, which could lead to changes in their habits, alter their lifestyle, their diet and social relationships. The objective of this review is to analysis of studies on fitness addiction in runners and establish the profile of the runner who is at risk of having an addiction to running. The search for articles was carried out in the Scopus andWeb of Science databases, using combinations of the keywords: «addiction», «physical exercise», «runners». The guidelines of the PRISMA statement were followed and the participants, instruments and results of the 12 selected studies were analyzed according to the inclusion / exclusion criteria, according to the relevance of the information extracted in the studies, specifying 5 evaluation criteria, evaluating the methodological rigor through a structured classification system. The main results indicate that runnorexia could occur in long-distance amateur runners between 35 and 45 years with less sports experience, with high Negative Running Addiction (NRA) and anxiety caused by the need to compete, seeking social recognition and dominance, worried about their mistakes and with high personal goals.
\end{abstract}

Key words: addiction; physical exercise; exercise; runners; run.

\section{Introducción}

Un número cada vez mayor de corredores parece ser vulnerable al Síndrome de Dependencia del Ejercicio (SDE), un trastorno clínico en el que el deportista no está dispuesto a alterar o suspender los regímenes de entrenamiento a pesar de las contraindicaciones médicas para el cumplimiento continuo del entrenamiento (Restrepo et al., 2021). Cuando un runner no puede estar varios días sin correr, cual síndrome de abstinencia, o no es capaz de descansar lo suficiente cuando tiene una lesión, hablamos de adicción al ejercicio físico. En esta revisión se hace referencia a la runnorexia o al runnerdependiente, un nuevo anglicismo que se ha implantado para dar nombre a esta adicción al running y

Fecha recepción: 19-03-21. Fecha de aceptación: 05-07-21

Joel Manuel Prieto Andreu

Joelmanuel.prieto@unir.net que no existe en las clasificaciones diagnósticas oficiales (Sanz, 2019). La runnorexia no es el mismo término que el sobreentrenamiento, la obsesión por correr puede llevar a un corredor a entrenar demasiado, lo que puede causar el sobreentreno. Sin embargo, cuando nos referimos a los síntomas que se producen en el organismo a corto plazo por no haber respetado el periodo de recuperación y de descanso, entonces sí que se puede decir que existe una relación entre runnorexia y sobreentrenamiento. Hay que diferenciar el deportista corredor de fondo del runner popular: el runner se autoentrena sin rigor técnico, no siendo consciente de los riesgos que puede tener una mala planificación en su salud, y el deportista sigue las indicaciones de su entrenador y/o equipo técnico para conseguir el mejor resultado posible, planificando y seleccionando sus competiciones. Para el runnorexico todo puede girar en torno al entrenamiento, lo que podría acarrear modificaciones en sus hábitos, alterando su estilo de vida e incluso 
su alimentación y relaciones sociales.

Siguiendo los principales resultados de la revisión de Nogueira, Salguero, y Márquez (2017) se indica que la adicción al deporte podría incluirse dentro de la categoría de los trastornos de la conducta, ya que cumple con la gran mayoría de los criterios diagnósticos y sobre todo por las graves consecuencias que esta puede ocasionar en el día a día de quienes la sufren. Glasser (1976) diferenció entre dos tipos de adicción a correr: la adicción positiva a correr (APC) como una actividad agradable causante de un gran placer, e incluso euforia, que provoca unos efectos mentales que hacen que la práctica sea tan placentera como adictiva, a estos efectos mentales Kostrubala (1977) les llamó euforia del corredor, explicando que se experimenta corriendo regularmente al menos durante cuarenta minutos seguidos. Stoll (1997) hipotetizó el fenómeno runners' high como la sensación de euforia que sienten los corredores tras una intensa carrera y que atribuyó a los efectos de las endorfinas. El concepto de APC se equiparó al del compromiso a correr (CC) (Carmack y Martens, 1979) en una investigación donde se desarrolló su instrumento de medida, la Commitment to Running Scale (CR). Por otro lado, Glasser (1976) definió la adicción negativa a correr (ANC) como aquella que domina la vida de una persona, de manera que llega a tener una actitud compulsiva, centrando su vida en salir a correr o competir en carreras, terminando por tomar decisiones que afectan al resto de su familia. Ogden Veale y Summers (1997) y Hausenblas y Downs (2002), al validar el Exercise Dependence Questionnaire (EDQ) y el Exercise Dependence Scale (EDS), respectivamente, obtuvieron tanto dimensiones positivas (gratificación positiva y efectos deseados) como negativas (los síntomas de abstinencia). Por otro lado, Morgan (1979) acuñó el término de «adicción negativa», para referirse a los efectos tanto a nivel fisiológico (incrementando la vulnerabilidad de sufrir algún tipo de lesión) como psicológico (alteraciones de la conducta), además de afectar a nivel social, familiar y laboral.

Existen diversas teorías explicativas de adicción al ejercicio físico, la hipótesis de evaluación cognitiva (Szabo, 1995), el Modelo de Cuatro Fases para la Adicción al Ejercicio (Freimuth, Moniz y Kim, 2011), el modelo biopsicosocial (McNamara y McCabe, 2012), o el modelo interaccional (Egorov y Szabo, 2013), aunque la mayoría de ellas coinciden en que esta adicción se debe tanto a factores de tipo fisiológico (la acción de las catecolaminas, la regulación de la Interlucina-6), como a factores de tipo psicológico derivados de los anterio- res (mejora del estado de ánimo, de la autoestima, confianza y relaciones sociales) que se producen con la práctica deportiva regular.

Dentro de los aspectos que influyen en la adicción al ejercicio físico, factores sociales (cultura, presión social por la imagen o estética) y factores biológicos (sustancias químicas, hormonas), destacan los aspectos psicológicos, entre ellos, los factores conductuales y comportamentales que obligan al runner a salir a correr de forma adictiva. En el estudio de Zandonai et al. (2020) indican que la dependencia del ejercicio es un fenómeno caracterizado por síntomas conductuales, fisiológicos y psicológicos de manera similar a los trastornos por uso de sustancias, siendo muchos los factores que contribuyen como la neuroadaptación a la exposición prolongada al ejercicio, las características del corredor y los factores contextuales. En estudio de Prieto (2019) las variables personales, como el trabajo, los hijos o el nivel de estudios, también se relacionaron con variables psicológicas como la ansiedad o el estrés, con la finalidad de establecer posibles relaciones que ayudasen a entender mejor el desempeño en la carrera a pie.

Es importante que el corredor aprenda a detectar estos síntomas para ayudarle a reconocer que su práctica deportiva ha podido dejar de ser una práctica de disfrute o de una pasión motivante, convirtiéndose en una práctica de carrera esclavizada y obsesiva que le produce angustia y desasosiego. En este sentido, De la Vega et al. (2016) encontraron que la pasión y la dedicación obsesiva a los deportes son buenos predictores de la dependencia al ejercicio. Esta obsesión, como cualquier otra adicción que domina la conducta de la persona, repercute en la salud mental del corredor, afectando también a su salud física, ya que se expone al cuerpo a un rendimiento exhaustivo por encima de la fatiga o de las lesiones deportivas.

En esta revisión se propone como objetivo principal establecer el perfil del corredor que se encuentra en riesgo de poseer una adicción a salir a correr. En esta línea, una investigación llevada a cabo por Martin et al. (2017) ha puesto en relieve el hecho de que las personas que practican deportes de resistencia continúan a pesar de estar lesionadas y además tienen altas puntuaciones en el Inventario de Adicciones al Ejercicio. Según Márquez y de la Vega (2015) la adicción a correr se caracteriza especialmente por la aparición de síntomas de abstinencia sufridos durante los períodos de privación (ansiedad, tensión, irritabilidad, espasmos musculares, etc.). Por otra parte, se especifican los objetivos específicos de la revisión: 
-Conocer la relación entre género, edad y experiencia deportiva con la adicción a correr.

-Conocer la relación entre volumen de práctica deportiva y adicción a correr.

-Conocer la relación entre hábitos de entrenamiento y adicción a correr.

-Determinar el riesgo de adicción a correr según distancia y variables psicológicas.

\section{Método}

Este estudio siguió las directrices de la declaración PRISMA para revisiones sistemáticas con el fin de estructurar el desarrollo correcto del documento (Hutton et al., 2015), tratándose de una revisión descriptiva con enfoque cualitativo.

\section{Estrategia de búsqueda y procedimiento}

Se han tenido en cuenta artículos que pasaran por una revisión por pares, publicados desde 2016 hasta 2021 por considerarse fuentes actuales, en todos los contextos geográficos, y escritos en español y en inglés en la Web of Science y Scopus. Las palabras clave utilizadas para la búsqueda fueron: addiction, physical exercise y runners. Para la selección de los estudios se han tenido en cuenta como criterios de exclusión los siguientes: comunicaciones a Congresos; artículos que estudien la adicción en otro tipo de deporte; publicaciones como artículos de revistas de divulgación, tesis o presentaciones en eventos académicos; trabajos pertenecientes a repositorios institucionales; trabajos procedentes de fuentes académicas no confiables. En cuanto a los criterios de inclusión se seleccionaron trabajos publicados del año 2016 en adelante, escritos en español y en inglés, y relacionados con la temática tras la lectura de su resumen y texto íntegro. En la última fase de inclusión de artículos para la revisión, se seleccionaron 12 trabajos como se puede observar en el fluxograma de la Figura 1.

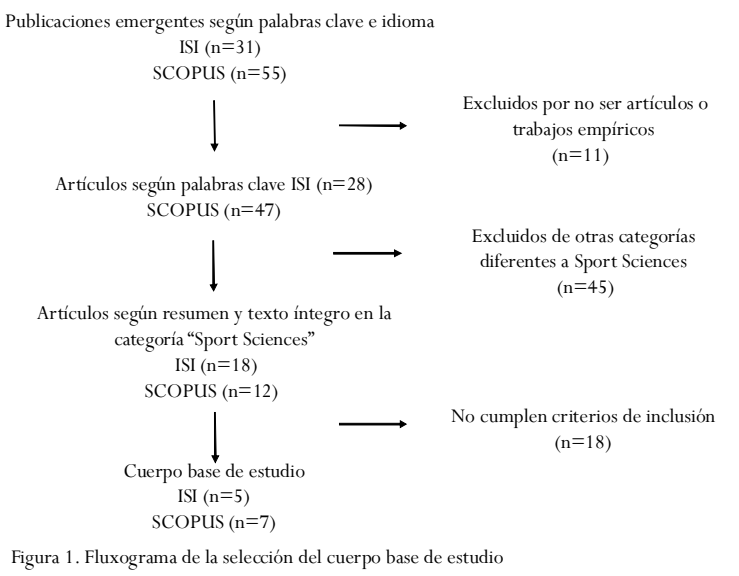

\section{Evaluación de la producción científica}

De acuerdo con la relevancia de la información extraída en los estudios, se concretan 5 indicadores de evaluación siguiendo la siguiente escala de valoración: muy relevante (5), relevante (3), poco relevante (1). Número de veces citado (I1): La media de citas de la muestra seleccionada fue de 10.92, se recodificó en distinta variable, estableciendo los siguientes puntos de corte: más de 10 citas (5), entre 4 y 9 citas (3) y menos de 3 citas (1); Coherencia metodológica (I2): califica la metodología utilizada en el estudio, si es clara, concluyente y con un alcance determinado; Aporte y replicabilidad (I3): cómo aporta el trabajo a la temática y su replicabilidad en contextos similares; Claridad argumentativa (I4): claridad de los argumentos presentados por el autor; Rigor metodológico (I5): el rigor metodológico y la calidad de los artículos (I5) se evaluó mediante un sistema de clasificación estructurado, verificado a través de la utilización de 11 criterios de calidad extraídos de Petticrew y Roberts (2006) y adaptado por Gast et al. (2017). Más de 8 criterios (5); entre 5 y 8 criterios (3); menos de 5 criterios (1).

\section{Resultados}

Se analiza el cuerpo base del estudio siguiendo los cinco indicadores de evaluación planteados: número de veces citado (I1), copherencia metodológica (I2), aporte y replicabilidad (I3), claridad argumentativa (I4) y rigor metodológico (I5).

En cuanto al indicador I1, el artículo más citado fue una revisión y tuvo 39 citas, respecto al resto de estudios de la misma temática, la media representa solamente un alcance del 35\%, por tanto, la difusión de los estudios incluidos en la revisión ha sido baja.

El indicador I2 sobre coherencia metodológica estima un $92 \%$ de valoración media, este porcentaje es muy relevante y relaciona coherentemente el título, el resumen y el encabezamiento con los objetivos de los estudios de la revisión, además se permite comprobar que los instrumentos utilizados respecto al objetivo de estudio son relevantes y coherentes.

La valoración del indicador I3 sobre el aporte del trabajo a la temática y su replicabilidad en contextos similares estima un $83 \%$, por lo que se puede considerar que tanto el objetivo como la fundamentación teórica de los artículos permiten identificar su cohesión y capacidad de volver a ser replicados.

El indicador I4 sobre claridad argumentativa arroja un resultado de un 90\%, lo que confirma que se detecta 
Tabla 1.

Artículos y revisiones incluidos en la revisión sistemática

\begin{tabular}{llccc}
\hline ID Año & Título & Participantes & Instrumentos & Conclusiones \\
\hline 1 & 2016 Dependencia al & 126 hombres (M $=$ & - Body Shape Questionnaire (BSQ) de Raich, & -La prevalencia de dependencia al ejercicio físico (DEF) fue de un 13.6\% y de insatisfacción corporal (IC) 22
\end{tabular} $\begin{array}{llll}\text { Dependencia al } & 126 \text { hombres ( } M= & \text { - Body Shape Questionnaire (BSQ) de Raich, } & \text {-La prevalencia } \\ \text { ejercicio físico e } & 36.02 \text { ) y } 23 \text { mujeres (M Mora, Soler, Avila, Clos y Zapater (1996). } & \text { de un } 16.3 \%\end{array}$

insatisfacción corporal $=28.18)$ que practican: - Escala Revisada de Dependencia del Ejercicio $\quad$-Los resultados obtenidos en este estudio señalan que determinadas motivaciones hacia el ejercicio físico en diferentes deportes carrera de fondo, _ (EDS-R) de Sicilia y González-Cutre (2011). se asocian con la DEF e IC.

de resistencia y su natación, triatlón y -Cuestionario de la Regulación de la Conducta en-Los triatletas presentan mayor prevalencia de dependencia al ejercicio físico, con diferencias significativas relación con la ciclismo el Ejercicio (BREQ-3) de Gonźlez-Cutre, Sicilia en relación con los corredores. motivación al deporte y Fernández (2010).

22016 Dependencia al 1795 corredores de $\quad$-Commitment to Running Scale-11 (CR-11). $\quad$-Los varones hacen un número de kilómetros significativamente más alto que las mujeres y llevan más 8 entrenamiento y fondo en ruta, 1541 -Running Addiction Scale-8 (RAS-8). años corriendo que ellas, de ahí, su elevado compromiso.

competición en hombres $(M=38.98)$ y -Hábitos de entrenamiento. $\quad$-Si el kilometraje es bajo, tanto en hombres como en mujeres, se incrementa un sano compromiso,

corredores de fondo 254 mujeres $(\mathrm{M}=\quad$ mientras que, si es alto, en hombres se incrementa su adicción negativa.

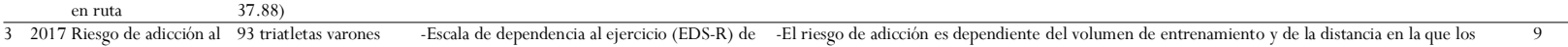
ejercicio en triatletas amateur (34.5 años) Sicilia y González-Cutre (2011). triatletas compiten, alcanzando una prevalencia del $30.0 \%$ en el caso de los triatletas de larga distancia. $\begin{array}{lll}\begin{array}{l}\text { ejercicio en triatletas } \\ \text { hombres amateur }\end{array} & \text { Sicilia y González-Cutre (2011). } & \text { triatletas compiten, alcanzando una prevalencia del } 30.0 \% \text { en el caso de los triatletas de larga distancia. } \\ & \text {-Cuestionario ad hoc. } & \text {-Existe prevalencia de riesgo de adicción en triatletas amateur (8.6\%), siendo este valor mayor que el de }\end{array}$ hombres amateur varones y su relacion -Existe prevalencia de riesgo de adicción en triatletas
otras poblaciones analizadas en la literatura científica. consente

2017 Adicción a correr: una 40 estudios analizados - Running Addiction Scale-8 (RAS-8). revisión desde sus que utilizan una muestra - Commitment to Running Scale-11 (CR-11 inicios hasta la de corredores o la -Escala de Motivación de los Maratonianos actualidad adicción como objeto de (MOMS-34) de Masters et al. (1993). estudio -La adicción al deporte podría incluirse dentro de la categoría de los trastornos de la conducta, estando $\quad 15$ asociado al constructo teórico del compromiso a correr y que incluye como variables de estimación el número de días de entrenamiento y el número de kilómetros semanales. -Los maratonianos son los corredores que mayores niveles de compromiso han reflejado, éste se convierte

\begin{tabular}{ll} 
estudio & en adicción negativa a correr (ANC) cuando dedican su día a día a entrenar. \\
\hline 5018 Exercise Addiction in 25 estudios analizados, 6 -Inventario de Adicciones del Ejercicio (IAE) de & -Los estudios revisados confirmaron que la práctica excesiva podría fomentar la aparición de conductas 39
\end{tabular} Practitioners of emplearon el IAE, 3 el Terry et al. (2004). adictivas y problemas de salud.

Endurance Sports: A MOMS, 3 la EDE, y el -Escala de Motivaciones de los maratonianos -La mayoría de las investigaciones no ha reportado ninguna edad o diferencias sexuales en la dependencia Literature Review resto combinaciones (MOMS) de Masters et al. (1993). del ejercicio entre los practicantes del deporte de resistencia; sin embargo, la pasión obsesiva y la incluyendo el RAS y el -Escala de Dependencia del Ejercicio (EDE) de dedicación a las actividades deportivas pueden ser predictores de la adicción al ejercicio. $\begin{array}{lll}\text { incluyendo el RAS y el } & \text {-Escala de Dependencia del Ejercicio (EDE) de dedicacion a las actividades deportivas pueden ser predictores de la adiccion al ejercicio. } \\ \text { CR. } & \text { Hausenblas y Downs (2002). } & \text {-El tipo de deporte más estudiado fue el de larga distancia (28\%), seguido de maratón (20\%) y triatlón }\end{array}$

\begin{tabular}{llll}
\hline 2019 & Exercise addiction and 257 corredores (48.9\% & -Escala de Dependencia del Ejercicio (EDS). & -Los resultados mostraron cinco variables que predijeron significativamente el riesgo de EA: ansiedad,
\end{tabular} its related factors in mujeres, $\mathrm{M}=40.49 \quad$-Escala de satisfacción general de la vida (GSLS). soledad, tiempo semanal dedicado a correr, actividad física realizada en la infancia y nivel educativo. amateur runners años). Un $53.6 \%$ de -Escala de Soledad (UCLA). -La soledad y la ansiedad pueden conducir a la abstinencia y a un comportamiento incontrolado que a su corredores con adicción -Cuestionario para medir la depresión, ansiedad vez conduce a una mayor cantidad de ejercicio en corredores aficionados. al ejercicio físico, un y estrés (DASS-21). $\quad$-Un nivel educativo bajo es un riesgo probable de desarrollo de EA. Es probable que estudiar en $37.8 \%$ no adictos y un -Subescala de imagen corporal del Body universidades mejore habilidades para lidiar con la angustia emocional y los mecanismos de afrontamiento $8,6 \%$ con prevalencia de Investment Scale (BIS). -La participación en actividades deportivas durante la infancia predice la probabilidad de EA.

riesgo de adicción. - Cuestionario para la medición de desórdenes

72019 Perfil psicológico en 61 corredores de $\quad$-Cuestionario de contenido de los objetivos en el -La dependencia del ejercicio se asoció con la motivación intrínseca para algunos factores y con la 9 $\begin{array}{lll}\text { corredores de Ultramaratón extrínseca para todos ellos. } & \text { ejercicio (GCEQ). }\end{array}$

$\begin{array}{llll}\text { ultramaratón } & (\mathrm{M}=40.09) \text { y } 5 & \text {-Escala Revisada de Dependencia del Ejercicio } & \text { extrinseca para todos ellos. } \\ & \text {-En este trabajo se encontraron relaciones significativas entre el factor "tolerancia" de dependencia del }\end{array}$ corredoras ( $M=36.11), \quad$ (ED-R). $\begin{array}{ll}\text { corredoras }(\mathrm{M}=36.11) \text {, } & \text { (ED-R). } \\ \text {-Cuestionario de Regulación de Conducta en el con los tres subtipos de motivación intrínseca: falta de contro, } \\ \text { conocimiento y motivación intrínseca de conocimiento y consecución }\end{array}$

$\begin{array}{ll}\text { Deporte (BRSQ). } & \text {-A mayor motivación intrínseca, menor práctica en relación a la planeada, disminuyendo el riesgo de }\end{array}$ -A mayor motivación intrínseca, menor prá
dependencia del ejercicio en este sentido.

82019 Adicción al atletismo 401 deportistas $\quad$ Escala de Adicción al Entrenamiento (EAE) $\quad$-Se concluyó que lo recomendable sería entrenar en un reducido grupo de compañeros que colaboren 3 en veteranos: un veteranos de atletismo enadaptada de la Escala de Adicción General (EAG) entre sí, sin la exigencia competitiva de un entrenador.

$\begin{array}{llll}\text { estudio con variables pista. } & \text { de Arbinaga y Caracuel (2005). Los valores más altos fueron obtenidos por los veteranos en la subescala placer y relajación, relacionada }\end{array}$

psicológicas y hábitos -Cuestionario de Satisfacción Intrínseca en el con los sentimientos positivos que se producen por entrenar. Sin embargo, también fueron altos los de entrenamiento Deporte, adaptada del Sport Satisfaction valores obtenidos en dos de las dimensiones no deseables de la adicción: carencia de control y tolerancia. Instrument (SSI) de Duda y Nicholls (1992). Todos estos datos, pueden quedar explicados con los valores tan altos obtenidos en la subescala diversión, -Cuestionario de Percepción de Éxito, adaptado que hacen que a estos deportistas les cueste abandonar o resistirse a iniciar sus entrenamientos o deseen del Perception of Success Questionnaire (POSQ) prolongarlos.

de Roberts y Balagué (1991).

de Roberts y Balague (19́1).
-Inventario de Percepción de las Creencias sobre

-Inventario de Percepción de las Creencias sobre
las Causas del Éxito en el Deporte, adaptada del las Causas del Exito en el Deporte, adaptada
Beliefs About the Causes of Sport Success Beliefs About the Causes of Sport Success
Questionnaire (BACSSQ) de Duda y Nicholls Question
(1992).

-Cuestionario sobre hábitos de entrenamiento

92019 Risk of Dependence 225 triatletas, $145 \quad$-Escala Revisada de Dependencia del Ejercicio on Sport in Relation tohombres $(\mathrm{M}=35.57)$ y (EDS-R). Body Dissatisfaction 80 mujeres ( $M=32.83$ ) $\quad$-Body Shape Questionnaire (BSQ).

-El 8.5\% de los sujetos tenían riesgo de dependencia del ejercicio y el $18.2 \%$ tienden a tener insatisfaccion corporal, sin diferencias significativas en el tipo de depor te que practican. -Hubo diferencias importantes con respecto a la dependencia del ejercicio fisico (15\% frente a $4.8 \%$ ) y la - Cuestionario de la Regulación de la Conducta eninsatisfacción corporal (31.1\% frente a $11 \%)$ en relación con el sexo, siendo el mayor porcentaje referido el Ejercicio (BREQ-3).

-Behavioural Aspect of the Body Image (BIAQ) -La dependencia al ejercicio físico (DPE), la imagen corporal (BI) y la restricción alimentaria se

para analizar las conductas de evitación de la $\quad$ correlacionan entre sí, siendo variables predictoras de la DPE.

imagen corporal.

102020 A descriptive study of 229 corredores italianos -Escala de dependencia del ejercicio con 21 ítems exercise dependence: ay 198 japoneses
(EDS-R). short report among Italian and Japanese -Cuestionario para evaluar la dependencia del runners ejercicio.

-Se realizó un estudio descriptivo de la dependencia del ejercicio en dos países diferentes, Italia y por
primera vez en Japón. En Italia, el $86,9 \%$ de los sujetos eran sintomáticos no dependientes, el 4,4\%

dependientes del ejercicio y el 8,7\% eran asintomáticos no dependientes. En Japón, los participantes se clasificaron como sintomáticos no dependientes $(49 \%)$ y asintomáticos no dependientes $(51 \%)$, pero ninguno se consideró en riesgo.

-Se encontraron diferencias significativas en las siete categorias de EDS-R entre las dos cohortes. -La dependencia del ejercicio es un complejo con muchos factores que contribuyen, como la neuroadaptación a la exposición prolongada al ejercicio, las características del corredor y los factores contextuales.

-El papel de los factores sociales y culturales de la dependencia del ejercicio necesitaría más investigaciones.

112021 Exercise Addiction in 513 corredores de fondo -La versión en español de la Escala de Adicción al -El objetivo de este estudio fue analizar el riesgo de adicción al ejercicio entre los corredores,

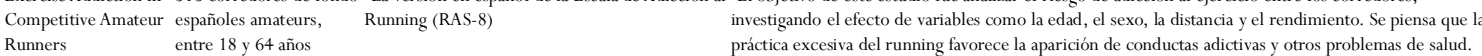
practica excesiva del running favorece la aparicion de conductas adictivas y otros problemas de salud,
-Los resultados generales mostraron que estos corredores presentan un riesgo mínimo de adicción, -Los resultados generales mostraron que estos corredores presentan un riesgo mínimo de adicción,
aunque la mayor edad y la mayor distancia habitual de carrera fueron factores de riesgo estadísticamente
significativos. significativos.

122021 Exercise Addiction in 248 corredores de larga -El historial de entrenamiento Long Distance distancia a partir de 18 -La Escala de medición de identidad atlética Runners
años de edad
(AIMS) de 10 items. -El propósito de este estudio fue identificar qué factores psicosociales y conductuales son más capaces de 4 predecir la adicción al ejercicio en los corredores. -La Escala de adicción al running (RAS) de 11 -Las variables predictoras significativas fueron el número de carreras realizadas por semana $(\mathrm{p}<0,001)$ y items

el número de lesiones en los 2 años anteriores $(\mathrm{p}<0,05)$. - Este estudio muestra que las puntuaciones más altas en el RAS se asociaron con una mayor identidad como deportista, más lesiones y más carreras por semana. --Aunque no fue una relación estadísticamente significativa, el índice de masa corporal $(\mathrm{p}=$ $0,084)$ y el número de años corriendo $(\mathrm{p}=0,098)$ se relacionaron negativamente con la puntuación RAS, lo que indica la necesidad de una muestra más amplia y diversa de corredores. 
con facilidad el problema planteado y nos permite constatar que los artículos seleccionados clarifican la calidad de los argumentos presentados en relación a los resultados y conclusiones y que, además, especifican tantos los participantes como los procedimientos en las conclusiones y/o discusión.

El indicador I5 sobre el rigor metodológico arroja un $77 \%$ que nos indica que la metodología o diseño de investigación utilizados en los estudios no son estadísticamente claros ni concluyentes debido a la diversidad de diseños aplicados en las investigaciones.

El objetivo de la revisión sistemática es realizar un análisis de los estudios sobre adicción al ejercicio físico en corredores, desde su indexación en las bases de datos ISI y Scopus desde la primera publicación indexada sobre la temática en el año 2016 hasta el año 2021. En la tabla 1, se han analizado los siguientes indicadores en los que se ha centrado la revisión sistemática de las 12 producciones científicas (10 artículos y 2 revisiones, 6 en español y 6 en inglés): participantes, instrumentos y principales conclusiones.

\section{Estado actual de la cuestión y discusión}

\section{Género, edad y experiencia deportiva}

Siguiendo a Latorre et al. (2016) variables como el sexo, la edad y llevar entrenando más de 12 años también son predictores de la Dependencia al Ejercicio Físico (DEF). En la misma línea, Simón-Grima (2020) indica que la adicción al ejercicio físico parece estar relacionada con mayores niveles de ansiedad-rasgo y con una menor edad. Del mismo modo, en el estudio de López et al. (2021) señalan que la edad y la distancia habitual de carrera fueron factores de riesgo estadísticamente significativos. En contraposición, en el estudio de Buck, Spittler, Reed y Khodaee (2018), no encontraron relaciones significativas entre el riesgo de dependencia del ejercicio y la edad. Por otra parte, una variable que ha resultado decisiva moderando la ANC y elevando el CC, es el mayor número de años de entrenamiento, puesto que la mayor experiencia de los corredores hace que tengan una dedicación más adecuada y saludable. En la misma línea, en el estudio de Prieto (2017) los corredores con menor experiencia deportiva presentaban mayor ansiedad cognitiva, siendo una característica que podría predecir la adicción al ejercicio físico. Cabe destacar que, aunque no fue una relación estadísticamente significativa, en el estudio de Maceri et al. (2021) el mayor número de años corriendo se relacionó con una mayor adicción al ejercicio físico.
Respecto a las diferencias de género en la adicción al ejercicio físico, siguiendo el estudio de Ruiz-Juan, Zarauz y Flores-Allende (2016) las mujeres, actualmente, emplean más tiempo diario y semanal para entrenar que los varones de todas las investigaciones precedentes, ya sea en la población de corredores de medio maratón o de maratón. Sin embargo, en el mismo estudio los varones hicieron un número de kilómetros significativamente más alto que las mujeres y llevan más años corriendo que ellas. Por otro lado, como indican Ruiz-Juan, Zarauz y Arufe-Giraldez (2019) ni en la adicción global, ni en ninguna de sus dimensiones, se han obtenido diferencias significativas por sexo, al igual que sucedía en las poblaciones analizadas por GonzálezCutre y Sicilia (2012). A pesar de las diferencias en los motivos de participación en carreras, las mujeres suelen correr más debido a una preocupación por controlar el peso y la imagen corporal, mientras que los hombres lo hacen debido al impacto de una naturaleza social y competitiva (Ertl et al., 2017, citado en Nogueira, Molinero, Salguero y Márquez, 2018), encontrándose diferencias únicamente en dos estudios. Por un lado, en el estudio de Pierce et al. (1997, citado en Nogueira, Molinero, Salguero y Márquez, 2018) con un pequeño grupo de corredores de maratón, encontraron que las mujeres obtuvieron una puntuación significativamente mayor en la dependencia del ejercicio que los hombres. Por otro lado, en el estudio de Torstveit, Fahrenholtz, Lichtenstein, Stenqvist y Melin (2019) hubo diferencias importantes con respecto a la dependencia del ejercicio físico (15\% frente a $4.8 \%$ ) y la insatisfacción corporal (31.1\% frente a 11\%) en relación con el sexo, siendo el mayor porcentaje referido a las mujeres.

\section{Volumen de práctica deportiva}

A nivel general, la práctica de actividad física se relaciona con una imagen corporal positiva, de modo que las personas más activas tienen una actitud más positiva hacia su propio cuerpo que los sujetos sedentarios. Sin embargo, la práctica de actividad física en exceso puede acarrear consecuencias negativas. Tras el análisis de los artículos de la presente revisión se han encontrado diferentes variables predictoras de la dependencia al ejercicio físico. En la misma línea, tal y como indican Lu et al., (2012, citado en Latorre et al., 2016), existe una asociación entre el nivel de práctica de actividad física y la DEF. Cabe destacar que en el estudio de Maceri et al. (2021) las variables predictoras con relaciones significativas con la adicción al ejercicio físico fueron el número de carreras realizadas por semana y también el número 
de lesiones en los 2 años anteriores.

Downs, Savage y Dinallo (2013) encontraron que la mayoría de jóvenes físicamente activos estaban dentro del rango sintomático, lo que sugiere que muchos adolescentes muestran signos tempranos de adicción al ejercicio físico, lo que puede conducir al desarrollo de adicción al ejercicio físico (EA) más tarde en la vida adulta entre la población físicamente activa. En la misma línea, en el estudio de Lukács, Sasvari, Varga y Mayer (2019) los resultados mostraron que la participación en actividades deportivas durante la infancia predice la probabilidad de EA.

En el estudio de Salas et al. (2013, citado en Latorre et al., 2016) encontraron que más de 4 sesiones semanales de práctica de carrera en relación a menos de 4 , arrojaba diferencias significativas en la dependencia negativa a la carrera en deportistas veteranos. Sin embargo, en el estudio de Latorre et al. (2016), la variable «estar entrenando más de 12 años» se convierte en el único predictor de la DEF relacionado con el entrenamiento. Por otro lado, Sachs (1981, citado en Ruiz-Juan et al., 2016) explicaba que los indeseables y negativos efectos de la ANC se manifiestan en los corredores a partir de 24-36 horas sin correr. Es de destacar que hacer ejercicio excesivamente no es necesariamente desadaptativo. Deportistas de élite realizan su entrenamiento con gran volumen e intensidad, pero no están en riesgo de EA. Si estuviesen en riesgo, podrían tener problemas para competir a un alto nivel, pero pueden existir deportistas de élite con algún tipo de adicción sin que ésta correlacione de forma negativa con el nivel competitivo alcanzado. Se debe puntualizar en que la adicción al ejercicio es una construcción compleja que examina los patrones de mala adaptación en relación con el comportamiento del ejercicio, si el deportista tiene la compulsión de hacer ejercicio en exceso, le podría causar perjuicios, no solo a su bienestar físico sino también a su bienestar psicológico. Por otro lado, los hallazgos del estudio de Lukács et al. (2019) respaldan la teoría de que los deportistas solitarios utilizan la actividad deportiva como fuente de alegría y felicidad. La lucha contra la ansiedad y la soledad puede hacer que los deportistas aumenten el tiempo o volumen de actividad deportiva, ya que necesitan cada vez más para lograr esa alegría y felicidad.

En cuanto a las diferencias entre corredores de medio maratón y maratón, según Ruiz-Juan et al. (2016) la menor ANC de los corredores de medio maratón respecto a los de maratón en estos últimos años, podría venir dada por la vía fisiológica, pues la dedicación ne- cesaria (en kilómetros y días a la semana de entrenamiento) para preparar un medio maratón es menor que la necesaria para entrenarse para un maratón, aunque la intensidad de los entrenamientos sí podría ser similar, siendo la exposición prolongada a un ejercicio intenso una variable a tener en cuenta en futuras investigaciones. En la misma línea, en una investigación en la que analizaban las diferencias entre los maratonistas con mayor y menor ANC, Ruiz-Juan y Zarauz (2012) obtuvieron que los que mostraron mayores valores de ANC hacían más kilómetros, entrenaban más días a la semana, dedicaban más horas al entrenamiento, y se preocupaban algo más por buscarse un entrenador y tener una mejor marca cercana a las 3 horas y media en los 6-7 maratones que han terminado. Por el contrario, los maratonistas con menor puntuación en ANC, tenían una menor preocupación por tener entrenador y era su primer maratón.

\section{Hábitos de entrenamiento}

Según Ruiz-Juan et al. (2016) los hábitos de entrenamiento de los deportistas pueden influir no sólo en su CC y ANC, sino en otros constructos psicológicos. En el estudio de Ruiz-Juan et al. (2016) recogieron información sobre hábitos de entrenamiento, promedio de kilómetros entrenados a la semana, tiempo por entrenamiento diario, días entrenados a la semana, porcentaje de tiempo que entrena solo a la semana, años corriendo, tener entrenador, compañeros de entrenamiento e índice de masa corporal (IMC). Por otro lado, en el estudio de Valenzuela y Arriba-Palomero (2017) abordaron los años de experiencia en el triatlón, su volumen de entrenamiento semanal medio en ese momento de la temporada (número de horas y sesiones por semana), y la distancia de mayor duración en la que normalmente competían (Sprint, Olímpica, Medio Ironman o Ironman). Por otra parte, en el estudio de Ruíz-Juan et al. (2019) se preguntaron sobre los siguientes hábitos de entrenamiento: ¿Cuál es su especialidad atlética habitual?, ¿A qué nivel ha llegado a competir en su mejor especialidad?, ¿Tiene entrenador?, ¿Cuánto tiempo entrena normalmente al día?, ¿Cuántos días entrena a la semana?, ¿Cuántos años lleva entrenando?, ¿Con cuántos deportistas entrena habitualmente?, ¿En cuántas competiciones participa al año?, sexo y edad. Para conocer y entender mejor los hábitos de entrenamiento y su relación con la adicción al ejercicio físico, en el estudio de Prieto (2016) sobre las razones que motivan a los deportistas a salir correr, se destacó que se hacen necesarias investigaciones que contribuyan a conocer y per- 
feccionar la orientación de los corredores.

\section{Riesgo de adicción según distancia y variables psicológicas}

Siguiendo a Nogueira et al. (2018) el tipo de deporte más estudiado fue el de larga distancia (28\%), seguido de maratón (20\%) y triatlón (8\%). En la misma línea, en esta revisión el deporte menos estudiado ha sido el triatlón. Según Nogueira et al. (2018) debido a la confusión terminológica y la variedad de herramientas utilizadas para medir la dependencia del ejercicio, las cifras de la prevalencia de este comportamiento difieren ampliamente entre los estudios, con valores citados que van del 3 al 42\% (Tornero Quiñones, Sáez Padilla, Castillo Viera, García Ferrete y Sierra Robles (2019).

En relación a los estudios con ultramaratonianos (corredores en carreras que superan la distancia de 42.195 kilómetros o las seis horas de duración), Buck et al. (2018) encontraron en su estudio que aproximadamente uno de cada cinco ultramaratonianos se encontraba «en riesgo» de adicción al ejercicio. En el estudio de Pierce, McGowan y Lynn (1993, citado en Guíu y Leyton, 2019), los ultramaratonianos presentaron puntuaciones de dependencia del ejercicio significativamente más altos que los de los corredores de maratón.

Por otro lado, los corredores del estudio de Latorre et al. (2016) presentan valores inferiores en el cuestionario Body Shape Questionnaire (BSQ) en comparación con los resultados del estudio de Fortes y Ferreira (2011, citado en Latorre et al., 2016), pero superiores en triatletas. Por otra parte, Torstveit et al. (2019), realizaron un estudio con deportistas varones de larga distancia, donde 20 eran corredores, los resultados mostraron que una mayor puntuación en la dependencia del ejercicio se asociaba con síntomas de desórdenes alimentarios.

En cuanto a las variables psicológicas, Schüler et al. (2018), con deportistas de ultra resistencia de los cuales 131 eran corredores, observaron que una pobre satisfacción de la necesidad básica de competencia se asociaba con la ansiedad, que predecía la dependencia del ejercicio. En este sentido, relacionando los tipos de ansiedad con la distancia, en el estudio de Prieto (2017) los corredores de larga distancia presentaron niveles más altos de ansiedad somática

Respecto a la motivación, en el estudio de Guíu y Leyton (2019) el factor «tolerancia» de dependencia del ejercicio se relacionó con los tres subtipos de motivación intrínseca. Según Buck, et al. (2018) este factor de tolerancia podría constituir simplemente una caracte- rística altamente prevalente de los corredores de ultramaratón en lugar de un indicador de dependencia.

En los corredores con mayor grado de adicción (committed runners), Thornton y Scott (1994, citado en Ruiz-Juan et al., 2019) obtuvieron que una mayor adicción a correr era debida a dos predictores; el reconocimiento social y el dominio. Por otro lado, Hamer et al. (2002, citado en Latorre et al., 2016) destacan a su vez que el más significativo predictor de la DEF es la regulación introyectada, seguido de la regulación identificada. Por otra parte, Leedy (2000, citado en RuizJuan et al., 2019) obtuvo que los corredores con mayor grado de adicción tenían niveles menores de ansiedad y depresión que los corredores clasificados como recreacionales.

Hall, Kerr, Kozub y Finnie (2007, citado en RuizJuan et al., 2019), obtuvieron que el 31\% del comportamiento de adicción al ejercicio podría explicarse por una combinación de orientaciones de meta de los deportistas, su percepción de competencia, la preocupación acerca de sus errores y unos altos estándares personales. Por otro lado, Ruiz-Juan et al. (2016) coinciden en que el compromiso a correr (CC) es un constructo psicológico sano, deseable y positivo para sus practicantes. A la suma de un alto CC, alta ANC y altas motivaciones para correr, Zarauz y Ruiz-Juan (2012) la denominaron súper-adherencia.

\section{Limitaciones}

Se han encontrado pocos estudios relacionados con la temática lo que hace que la muestra de estudios analizados en la revisión no llegue a ser representativa. Tampoco se emplearon técnicas de metaanálisis y la evaluación de la producción científica fue realizada por un único evaluador. Por otro lado, en los trabajos revisados se evidenció que las investigaciones por lo general se hacen con muestras muy pequeñas y en tiempos muy cortos, lo cual se considera poco apropiado en el ámbito deportivo, donde se puede requerir comparar muestras más grandes en estudios longitudinales. Se sugiere que en futuras investigaciones se empleen muestras grandes para detectar diferencias significativas entre sujetos con o sin adicción a correr, cuando realmente éstas existan. Por otra parte, la investigación longitudinal aportaría los beneficios de tener marcos de tiempo extendidos, lo que permitiría establecer relaciones causales para conocer y entender mejor los factores adictivos en carrera continua. 


\section{Conclusiones de la revisión}

A la vista de los resultados obtenidos en los estudios analizados en la presente revisión (10 trabajos empíricos o estudios de investigación y 2 revisiones, 6 en inglés y 6 en español) aunque hay que reconocer las limitaciones metodológicas de los estudios en este campo, por la terminología y por lo expuesto en el apartado de limitaciones, se destacan las siguientes conclusiones:

-La mayor experiencia de los corredores modera la ANC y eleva el CC, haciendo que los deportistas tengan una dedicación más saludable y adecuada, siendo lo deseable ya que una alta ANC en carrera podría acarrear una actitud compulsiva, centrando su vida en salir a correr o competir en carreras.

-No hay diferencias significativas por sexo ni en la adicción global, ni en ninguna de sus dimensiones, solamente en los motivos de práctica. En este sentido, las mujeres son las que más suelen correr debido a una preocupación estética, por controlar el peso y por la imagen corporal, mientras que los hombres lo hacen por socializar y competir.

-Los expertos deben ser conscientes del potencial adictivo de las carreras de larga distancia y de la influencia que tiene el volumen de entrenamiento en la aparición de la adicción. Se considera un factor de riesgo competir en larga distancia y aumentar el volumen de entrenamiento, condición sine qua non.

-Los hábitos de entrenamiento de los deportistas como el promedio de kilómetros y días entrenados a la semana, el tiempo por entrenamiento diario, los años corriendo y tener entrenador o compañeros de entrenamiento pueden influir no sólo en su CC y ANC, sino en otros constructos psicológicos.

-Debido a la confusión terminológica y la variedad de herramientas utilizadas para medir la dependencia del ejercicio (Body Shape Questionnaire (BSQ), Escala Revisada de Dependencia del Ejercicio (EDS-R), Cuestionario de Regulación de la Conducta en el Ejercicio (BREQ-3), Inventario de Adicciones del Ejercicio (IAE), Escala de Dependencia del Ejercicio (EDE), Escala de Adicción al Entrenamiento (EAE), Escala de adicción al running (RAS) y la Escala de medición de identidad atlética (AIMS)), las cifras de la prevalencia de este comportamiento difieren ampliamente entre los estudios incluidos en la revisión, en la misma línea como indican Nogueira et al. (2018), con valores citados que van del 3 al $42 \%$.

- Tras la revisión de los estudios analizados los siguientes factores podrían predecir la dependencia del ejercicio: la ansiedad provocada por la necesidad de competir, la búsqueda de reconocimiento social y dominio, la preocupación acerca de sus errores y unos altos estándares personales.

Aunque hasta la fecha es difícil dar respuestas inequívocas a las razones reales del desarrollo del riesgo de adicción al ejercicio físico o a los posibles perfiles de riesgo, siguiendo los resultados de la presente revisión, la runnorexia podría presentarse en corredores aficionados con un nivel educativo bajo y una menor experiencia deportiva, y, que hayan practicado ejercicio físico durante la infancia. Asimismo, la runnorexia podría manifestarse en mujeres preocupadas por controlar el peso y la imagen corporal y en hombres de naturaleza competitiva, con elevada soledad y ANC, con elevada ansiedad provocada por la necesidad de competir, con búsqueda de reconocimiento social y dominio, con preocupación acerca de sus errores y con unos altos estándares personales. Por último, se destaca el rango de edad de 35 a 45 años, siendo diversos los motivos que se podrían plantear por un exceso de práctica adictiva hacia el ejercicio físico a estas edades: crisis de la mediana edad en el que el sujeto se cuestione la práctica deportiva como expectativa de vida, excesiva práctica deportiva para superar un divorcio o una ruptura, búsqueda de sensaciones y/o novedades en la vida, necesidad de sentir el placer de salir a correr al aire libre por sobreexposición a ambientes laborales cerrados o confinamientos como el ocurrido durante la pandemia de la COVID-19, falta de perspectiva vital, actividad compensatoria o deficitaria a nivel vital, Síndrome de Peter Pan por el cuál el sujeto inmaduro no se comporte como sus coetáneos y practique deporte para sentirse joven bajo el mantra «ahora o nunca», para sentirse seguros de sí mismos potenciando su autoestima, por establecer una interacción social por sentirse parte de un grupo, por el cumplimiento de logros de metas personales, por atracción hacia la competencia o por reconocimiento con la finalidad de que los seres queridos se sientan orgullosos.

La presente revisión aporta información útil sobre los posibles factores de adicción al running, ayudando a contribuir en la elaboración de programas psicológicos de entrenamiento. Los psicólogos y los profesionales del deporte y del ejercicio podrían ayudar a construir nuevos límites en la actividad del deportista, ayudándole a incorporar otros ámbitos de interés en su vida de forma progresiva. Los resultados de la revisión tienen aplicabilidad práctica y pueden servir de utilidad para entrenadores y psicólogos deportivos para detectar po- 
sibles perfiles potenciales de riesgo de adicción en deportistas que puedan presentar asociaciones con las variables estudiadas. Por último, se requieren estudios que analicen la adicción al ejercicio físico comparando volumen e intensidad de entrenamiento y, respecto a la distancia en carrera, comparaciones entre corredores de larga distancia, en ultramaratón y triatlón, abordando las diferencias entre sexos.

\section{Referencias}

Buck, K., Spittler, J., Reed, A. y Khodaee, M. (2018). Psychological Attributes of Ultramarathoners. Wilderness \& Environmental Medicine, 29, 66-71. doi: 10.1016/ j.wem.2017.09.003

Carmack, M. y Martens, R. (1979). Measuring commitment to running: A survey of runners' attitudes and mental states. Journal of Sport Psychology, 1,25-42. doi:10.1123/jsp.1.1.25

De La Vega, R., Parastatidou, I., Ruíz-Barquín, R., y Szabo, A. (2016). Exercise addiction in athletes and leisure exercisers: The moderating role of passion. Journal of Behavioral Addictions, 5(2), 325-331. doi:10.1556/2006.5.2016.043

Downs, D. S., Savage, J. S., y DiNallo, J. M. (2013). Selfdetermined to exercise? Leisure-time exercise behavior, exercise motivation, and exercise dependence in youth. Journal of Physical Activity and Health, 10(2), 176-184. doi:10.1123/jpah.10.2.176

Egorov, A.Y. y Szabo, A. (2013). The exercise paradox: an interactional model for a clearer conceptualization of exercise addiction. J. Behav.Addict. 2, 199-208. doi: 10.1556/JBA.2.2013.4.2

Freimuth, M., Moniz, S., y Kim, S. R. (2011). Clarifying exercise addiction: diûerential diagnosis, cooccurring disorders, and phases of addiction. Int. J. Environ. Res. Public Health 8, 4069-4081. doi:10.3390/ ijerph8104069

Gast, I., Schildkamp, K. y Van der Veen, J.T. (2017). Team-Based Professional Development Interventions in Higher Education: A Systematic Review. Review of Educational Research, 87(4), 736-767. doi:10.3102/ 0034654317704306

Glasser, W. (1976). Positive addiction. New York, Estados Unidos: Harper y Row

González-Cutre, D., y Sicilia, A. (2012b). Motivation and Exercise Dependence: A Study Based on SelfDetermination Theory. Research Quarterly for Exercise and Sport, 83, 318-329. doi:10.5641/ 027013612800745194
Guíu, M. y Leyton, M. (2019). Perfil psicológico en corredores de ultramaratón. Retos, 36(36), 310-317.

Hausenblas, H. A. y Downs, D. S. (2002). How much is too much? The development and validation of the exercise dependence scale. Psychol. Health 17, 387404. doi: 10.1080/0887044022000004894

Hutton, B., Salanti, G., Caldwell, D. M., Chaimani, A., Schmid, C. H., Cameron, C., Ioannidis, J. P. A., Straus, S., Thorlund, K., Jansen, J. P., Mulrow, C., Catalá-López, F., Gøtzsche, P. C., Dickersin, K., Boutron, I., Altman, D. A., y David Moher, D. (2015). The PRISMA extension statement for reporting of systematic reviews incorporating network metaanalyses of health care interventions: Checklist and explanations PRISMA extension for network metaanalysis. Annals of Internal Medicine, 162(11), 777784. doi: https://doi.org/10.7326/M14-2385

Kostrubala, T. (1977). The joy of running. New York: Simon \& Schuster

Latorre, P., Pinillos, F., Obra, A. y Montilla, J.A. (2016). Dependencia al ejercicio físico e insatisfacción corporal en diferentes deportes de resistencia y su relación con la motivación al deporte. Revista de Psicologia del Deporte. 25(1), 113-120.

López, A. N., del Valle, A. S., y Rosa, S. M. (2017). Adicción a correr: una revisión desde sus inicios hasta la actualidad. Revista de Psicología Aplicada al Deporte $y$ al Ejercicio Físico, 2(1), 6.

López, A. N., Salguero, A., Molinero, O., Rosado, A., \& Márquez, S. (2021). Exercise Addiction in Competitive Amateur Runners. International Journal of Mental Health and Addiction, 1-17.

Lukács, A., Sasvari, P., Varga, B., y Mayer, K. (2019). Exercise addiction and its related factors in amateur runners. Journal ofbehavioral addictions, 8(2), 343-349. doi:10.1556/2006.8.2019.28

Maceri, R. M., Cherup, N. P., Buckworth, J., y Hanson, N. J. (2021). Exercise addiction in long distance runners. International Journal of Mental Health and Addiction, 1-10.

Márquez, S. y de la Vega, R. (2015). La adicción al ejercicio: un trastorno emergente de la conducta. Nutrición Hospitalaria, 31, 2384-2391. doi:10.3305/ nh.2015.31.6.8934

Martin, L.E., Sisante, J.F., Wilson, D.R., Moody, A.A., Savage, C.R. y Billinger, S.A. (2017). Pilot study of endurance runners and brain responses associated with delay discounting. Int.J. Exerc. Sci. 10, 690-701.

McNamara, J., y McCabe, M. (2012). Striving for success or addiction? exercise dependence among elite 
australian athletes. J. Sports Sci. 30, 755-766. doi: 10.1080/02640414.2012.667879

Morgan,W. (1979). Negative addiction in runners. Phys. Sports Med. 7, 57-70. doi: 10.1080/ 00913847.1979 .11948436

Nogueira,A., Salguero, A. y Márquez, S. (2017). Adicción a correr: una revisión desde sus inicios hasta la actualidad. Revista de Psicología Aplicada al Deporte y al Ejercicio Físico, 1(6), 1-22. doi: 10.5093/rpadef2017a1

Nogueira, A., Molinero, O., Salguero, A. y Márquez, S. (2018). Exercise Addiction in Practitioners of Endurance Sports: A Literature Review. Frontiers in Psychology, 9, 1-11. doi: 10.3389/fpsyg.2018.01484

Ogden, J., Veale, D. y Summers, Z. (1997). The development and validation of the exercise dependence questionnaire. Addict. Res. 5, 343-355. doi: 10.3109/16066359709004348

Prieto, J.M. (2016). Razones para correr de corredores populares. Athlos: Revista internacional de ciencias sociales de la actividad física, el juego y el deporte, 11(11), 717.

Prieto, J.M. (2017). Experiencia deportiva, ansiedad y motivación en corredores populares. Cuadernos de Psicología del Deporte, 17(1), 51-58.

Prieto, J.M. (2019). Sensaciones y ansiedad que experimentan corredores populares y su relación con variables personales. Trances: Transmisión del conocimiento educativo y de la salud, 11(2), 305-324.

Restrepo, J. E., Quirama, T. C., y Montoya, G. P. C. (2021). Propiedades psicométricas de la Escala de Dependencia al Ejercicio-Revisada (EDS-R) en usuarios colombianos de gimnasios. Retos, 41, 782-790.

Ruiz-Juan F. y Zarauz, A. (2012). Variables que hacen adicto negativamente a correr al maratoniano español. Retos, 21, 38-42.

Ruiz-Juan, F., Zarauz, A. y Flores-Allende, G. (2016). Dependencia al entrenamiento y competición en corredores de fondo en ruta. Cultura_Ciencia_Deporte, 11(32), 149-155. doi:10.12800/ccd.v11i32.714

Ruiz-Juan, F., Zarauz, A. y Arufe-Giraldez, V. (2019). Adicción al atletismo en veteranos: un estudio con variables psicológicas y hábitos de entrenamiento. Retos, 35, 201-207.

Sanz, E. (2019). 'Runnorexia' o cuando la obsesión por el 'running' sobrepasa todos los límites [en línea]. Recuperado de: https://bit.ly/3he1k1d

Schüler, J., Knetchle, B. y Wegner, M. (2018). Antecedents of Exercise Dependence in UltraEndurance Sports: Reduced Basic Need Satisfaction and Avoidance Motivated Self-Control. Frontiers in
Psychology, 9(1275), 1-8. doi:10.3389/ fpsyg. 2018.01275

Simón-Grima, J., San Martín-Salvador, A., EstradaMarcén, N., y Casterad-Seral, J. (2020). Relación entre la adicción al ejercicio, el uso de dispositivos fitness y la ansiedad rasgo (Relationship between exercise addiction, use of fitness devices and trait anxiety). Retos, 39, 525-531. doi:10.47197/ retos.v0i39.80078

Stoll, O. (1997). Endorphine, Laufsucht und runner's high. Aufstieg und niedergang eines mythos [Endogenous opiates, «runner's high» and «Exercise Addiction» - the rise and decline of a myth]. Leipziger Sportwissenschaftliche Beitraege, 28(1), 102-121.

Szabo, A. (1995). The impact of exercise deprivation on well-being of habitual exercisers. Australian J.Sci. Med. Sport 27, 68-75.

Tornero Quiñones, I., Sáez Padilla, J., Castillo Viera, E., García Ferrete, J. J. y Sierra Robles, Á. (2019). Risk of Dependence on Sport in Relation to Body Dissatisfaction and Motivation. Sustainability, 11(19),1-14. doi:10.3390/su11195299

Torstveit, M., Fahrenholtz, I., Lichtenstein, M., Stenqvist, T. y Melin,A. (2019). Exercise dependence, eating disorder symptoms and biomarkers of Relative Energy Deficiency in Sports (RED-S) among male endurance athletes. BMJ Open Sport \& Exercise Medicine, 5, 1-8. doi: 10.1136/ bmjsem-2018000439

Valenzuela, P., y Arriba-Palomero, F. (2017). Riesgo de adicción al ejercicio en triatletas hombres amateur varones y su relación con variables de entrenamiento. RICYDE. Revista Internacional de Ciencias del Deporte, 13(48), 162-171. doi:10.5232/ricyde2017.04806

Zandonai,T., Manresa-Rocamora, A., Monese, L., MoyaRamón, M., Schena, F., y Chiamulera, C. (2020). A descriptive study of exercise dependence: a short report among Italian and Japanese runners. Journal of Addictive Diseases, 39(1), 133-137.

Zarauz, A. y Ruiz-Juan, F. (2012). «Súper-adherencia» del maratoniano; variables predictoras y diferencias de género. Universitas Psychologica, 11(3), 863-87. doi:10.11144/Javeriana.upsy11-3.smvp

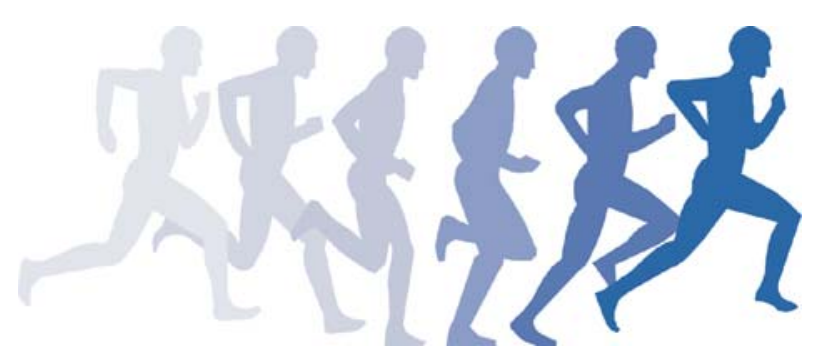

